\title{
Almost conformally flat hypersurfaces
}

\author{
Christos-Raent Onti and Theodoros Vlachos
}

\begin{abstract}
We prove a universal lower bound for the $L^{n / 2}$-norm of the Weyl tensor in terms of the Betti numbers for compact $n$-dimensional Riemannian manifolds that are conformally immersed as hypersurfaces in the Euclidean space. As a consequence, we determine the homology of almost conformally flat hypersurfaces. Furthermore, we provide a necessary condition for a compact Riemannian manifold to admit an isometric minimal immersion as a hypersurface in the round sphere and extend a result due to Shiohama and $\mathrm{Xu}[18]$ for compact hypersurfaces in any space form.
\end{abstract}

\section{Introduction}

The investigation of curvature and topology of Riemannian manifolds or submanifolds is one of the basic problems in global differential geometry. The sphere theorem is an important result in this direction, in the framework of Riemannian geometry.

In the realm of conformal geometry, the fundamental tensor is the Weyl tensor and his role is similar to the one of the curvature tensor in Riemannian geometry. Several authors have worked on the question how certain conditions on the Weyl tensor affect the geometry and the topology of Riemannian manifolds (cf. [3, 8]). Schouten's theorem asserts that the vanishing of the Weyl tensor of a Riemannian $n$-manifold $M^{n}$ is equivalent to the fact that $M^{n}$ is conformally flat, i.e., locally is conformally diffeomorphic to an open subset of the Euclidean space $\mathbb{R}^{n}$, with the canonical metric, if $n \geq 4$. The $L^{n / 2}$ norm of the Weyl tensor, which is a conformal invariant, measures how far a compact Riemannian manifold deviates from being conformally flat. There are plenty of papers that investigate the effect of restrictions on the $L^{n / 2}$-norm of the Weyl tensor to both geometric and topological properties (cf. [1,9,10,13,17,21]).

Cartan [2] initiated the investigation of conformally flat manifolds from the submanifold point of view. Moore [15] and later do Carmo, Dajczer and Mercuri [7] studied conformally flat submanifolds of the Euclidean space (see also $[6,16]$ ). In particular, in [7]

2010 Mathematics Subject Classification. Primary 53C40, 53C20; Secondary 53C42.

Key Words and Phrases. Weyl tensor, $L^{n / 2}$-norm of Weyl tensor, Betti numbers, conformal immersions. 
the authors studied the case of hypersurfaces in $\mathbb{R}^{n+1}$ and they proved that diffeomorphically every such hypersurface $M^{n}$ is a sphere $\mathbb{S}^{n}$ with $\beta_{1}\left(M^{n} ; \mathbb{Z}\right)$ handles attached, where $\beta_{1}\left(M^{n} ; \mathbb{Z}\right)$ is the first Betti number of $M^{n}$. Moreover, they showed that geometrically every such hypersurface locally consists of nonumbilic submanifolds of $\mathbb{R}^{n+1}$ that are foliated by complete round $(n-1)$-spheres and are joined through their boundaries to the following three types of umbilic submanifolds of $\mathbb{R}^{n+1}:(i)$ an open piece of an $n$-sphere or an $n$-plane bounded by round $(n-1)$-sphere, $(i i)$ a round $(n-1)$-sphere, $($ iii $)$ a point.

It is therefore natural to address the following

Question. Let $\left(M^{n}, g\right)$ be a compact $n$-dimensional Riemannian manifold that admits a conformal immersion in $\mathbb{R}^{n+1}$. What can be said about the topology of $M^{n}$ if $\left(M^{n}, g\right)$ is almost conformally flat, in the sense that the $L^{n / 2}$-norm of the Weyl tensor is sufficiently small?

The aim of the paper is to provide an answer to the above question. In particular, we give a universal lower bound for the $L^{n / 2}$-norm of the Weyl tensor in terms of the Betti numbers for compact $n$-dimensional Riemannian manifolds that are conformally immersed in $\mathbb{R}^{n+1}$. As a consequence, we are able to determine the homology of compact almost conformally flat hypersurfaces. Throughout the paper, all manifolds under consideration are assumed to be without boundary, connected and oriented. Our main result is stated as follows.

Theorem 1. Given $n \geq 4$, there exists a positive constant $c(n)$, depending only on $n$, such that if $\left(M^{n}, g\right)$ is a compact $n$-dimensional Riemannian manifold that admits a conformal immersion in $\mathbb{R}^{n+1}$, then the Weyl tensor associated to $g$ satisfies

$$
\int_{M^{n}}\left\|\mathcal{W}_{g}\right\|^{n / 2} d M_{g} \geq c(n) \sum_{i=2}^{n-2} \beta_{i}\left(M^{n} ; \mathbb{F}\right),
$$

where $\beta_{i}\left(M^{n} ; \mathbb{F}\right)$ is the $i$-th Betti number of $M^{n}$ over an arbitrary coefficient field $\mathbb{F}$. In particular, if

$$
\int_{M^{n}}\left\|\mathcal{W}_{g}\right\|^{n / 2} d M_{g}<c(n)
$$

then $M^{n}$ has the homotopy type of a $C W$-complex with no cells of dimension $i$ for $2 \leq$ $i \leq n-2$ and the fundamental group $\pi_{1}\left(M^{n}\right)$ is a free group on $\beta_{1}\left(M^{n} ; \mathbb{Z}\right)$ generators. Moreover, if $\pi_{1}\left(M^{n}\right)$ is finite then $M^{n}$ is homeomorphic to the sphere $\mathbb{S}^{n}$.

Thus, in the case where (2) is satisfied, the homology groups of $M^{n}$ must satisfy the condition $H_{i}\left(M^{n} ; \mathbb{F}\right)=0$ for all $2 \leq i \leq n-2$, where $\mathbb{F}$ is any coefficient field.

The assumption on the codimension in Theorem 1 is essential, as the following example shows. We consider the manifold $M^{n}=\mathbb{S}^{1}(1) \times \mathbb{S}^{1}(1) \times \mathbb{S}^{n-2}(r), n \geq 4$, equipped with the product metric $g$, where $\mathbb{S}^{n-2}(r)$ is the $(n-2)$-dimensional round sphere of radius 
$r$. Since $M^{n}$ is isometrically immersed into the sphere $\mathbb{S}^{n+2}\left(\sqrt{2+r^{2}}\right)$ in the obvious way, it follows that $M^{n}$ admits a conformal immersion into $\mathbb{R}^{n+2}$. A long but straightforward computation yields that

$$
\int_{M^{n}}\left\|\mathcal{W}_{g}\right\|^{n / 2} d M_{g}=\frac{a(n)}{r^{2}}
$$

where $a(n)$ is a positive constant depending only on $n$. Since the second Betti number of $M^{n}$ is non-zero, over an arbitrary coefficient field $\mathbb{F}$, it follows that $M^{n}$ does not satisfy inequality (1) for $r$ large enough.

Remark 2. In Theorem 1 , the ambient space $\mathbb{R}^{n+1}$ can be replaced by the round sphere $\mathbb{S}^{n+1}$ or the hyperbolic space $\mathbb{H}^{n+1}$. Indeed, this follows from the fact that $\mathbb{S}^{n+1} \backslash\{$ point and $\mathbb{H}^{n+1}$ are conformally equivalent to the Euclidean space and that the $L^{n / 2}$-norm of the Weyl tensor is conformally invariant.

Chern and Simons provided in [5, Theorem 6.4, p. 65] a necessary condition for a compact 3-dimensional Riemannian manifold to admit a conformal immersion into $\mathbb{R}^{4}$. Theorem 1 allows us to give another such condition.

Corollary 3. Let $\left(M^{n}, g\right), n \geq 4$, be a compact $n$-dimensional Riemannian manifold. A necessary condition that $M^{n}$ admit a conformal immersion in $\mathbb{R}^{n+1}$ is inequality (1).

There is an abundance of Riemannian manifolds that do not satisfy inequality (1), and therefore do not admit a conformal immersion as hypersurfaces in $\mathbb{R}^{n+1}$. For instance, let $M^{n}=N^{m} \times \mathbb{S}^{n-m}(r), \quad 2 \leq m \leq n-2$, equipped with the product metric $g$, where $N^{m}$ is a compact flat $m$-dimensional Riemannian manifold. A long but straightforward computation yields that

$$
\int_{M^{n}}\left\|\mathcal{W}_{g}\right\|^{n / 2} d M_{g}=\frac{a(n, m)}{r^{m}} \operatorname{Vol}\left(N^{m}\right),
$$

where $a(n, m)$ is a positive constant depending only on $n$ and $m$. Since, the $(n-m)$-th Betti number of $M^{n}$ is non-zero, over an arbitrary coefficient field $\mathbb{F}$, we obtain that $M^{n}$ does not satisfy (1) for $r$ large enough.

Using Theorem 1, we are able to prove the following result for compact minimal hypersurfaces in spheres.

Theorem 4. Given $n \geq 4$, there exists a positive constant $c_{1}(n)$, depending only on $n$, such that if $\left(M^{n}, g\right)$ is a compact $n$-dimensional Riemannian manifold that admits an isometric minimal immersion into the unit $(n+1)$-dimensional sphere $\mathbb{S}^{n+1}$, then

$$
\int_{M^{n}}\left\|\operatorname{Ric}_{g}-(n-1) g\right\|^{n / 2} d M_{g} \geq c_{1}(n) \sum_{i=2}^{n-2} \beta_{i}\left(M^{n} ; \mathbb{F}\right)
$$


where $\mathrm{Ric}_{g}$ is the Ricci tensor of $\left(M^{n}, g\right)$. In particular, if

$$
\int_{M^{n}}\left\|\operatorname{Ric}_{g}-(n-1) g\right\|^{n / 2} d M_{g}<c_{1}(n),
$$

then $M^{n}$ has the homotopy type of a $C W$-complex with no cells of dimension $i$ for $2 \leq$ $i \leq n-2$ and the fundamental group $\pi_{1}\left(M^{n}\right)$ is a free group on $\beta_{1}\left(M^{n} ; \mathbb{Z}\right)$ elements. Moreover, if $\pi_{1}\left(M^{n}\right)$ is finite then $M^{n}$ is homeomorphic to the sphere $\mathbb{S}^{n}$.

As an immediate application of Theorem 4, we obtain an obstruction for a Riemannian metric to be realized on a compact minimal hypersurface of the sphere.

Corollary 5. A compact $n$-dimensional Riemannian manifold $\left(M^{n}, g\right), n \geq 4$, that satisfies

$$
\int_{M^{n}}\left\|\operatorname{Ric}_{g}-(n-1) g\right\|^{n / 2} d M_{g}<c_{1}(n) \sum_{i=2}^{n-2} \beta_{i}\left(M^{n} ; \mathbb{F}\right),
$$

cannot admit an isometric minimal immersion into the unit sphere $\mathbb{S}^{n+1}$.

Shiohama and $\mathrm{Xu}[18]$ gave a lower bound in terms of the Betti numbers for the $L^{n / 2}$ norm of the $(0,4)$-tensor $R_{g}-\left(\operatorname{scal}_{g} / n(n-1)\right) R_{1}$ of compact hypersurfaces in $\mathbb{R}^{n+1}$. Here, $R_{g}$ and $\mathrm{scal}_{g}$ denote the $(0,4)$-curvature tensor and the scalar curvature of the induced metric $g$ respectively and $R_{1}=(1 / 2) g \otimes g$, where $\otimes$ stands for the Kulkarni-Nomizu product. The $L^{n / 2}$-norm of this tensor measures how far the Riemannian manifold deviates from being a space form. Their proof strongly uses the fact that the ambient space is the Euclidean one. Using Theorem 1, we extend their result for compact hypersurfaces in spheres or in the hyperbolic space.

Theorem 6. If a compact $n$-dimensional Riemannian manifold $\left(M^{n}, g\right), n \geq 4$, admits an isometric immersion into the sphere $\mathbb{S}^{n+1}$ or the hyperbolic space $\mathbb{H}^{n+1}$, then

$$
\int_{M^{n}}\left\|R_{g}-\frac{\operatorname{scal}_{g}}{n(n-1)} R_{1}\right\|^{n / 2} d M_{g} \geq c(n) \sum_{i=2}^{n-2} \beta_{i}\left(M^{n} ; \mathbb{F}\right),
$$

where $c(n)$ is the constant that appears in Theorem 1. In particular, if

$$
\int_{M^{n}}\left\|R_{g}-\frac{\mathrm{scal}_{g}}{n(n-1)} R_{1}\right\|^{n / 2} d M_{g}<c(n),
$$

then $M^{n}$ has the homotopy type of a $C W$-complex with no cells of dimension $i$ for $2 \leq$ $i \leq n-2$ and the fundamental group $\pi_{1}\left(M^{n}\right)$ is a free group on $\beta_{1}\left(M^{n} ; \mathbb{Z}\right)$ elements. Moreover, if $\pi_{1}\left(M^{n}\right)$ is finite then $M^{n}$ is homeomorphic to the sphere $\mathbb{S}^{n}$.

We notice that related results have been obtained in [19, 20,22]. 


\section{$2 \quad$ Algebraic auxiliary results}

This section is devoted to some algebraic results that are crucial for the proofs. Let $V$ be a finite dimensional real vector space equipped with a positive definite inner product $\langle\cdot, \cdot\rangle$. We denote by $\operatorname{Hom}(V \times V, \mathbb{R})$ the space of all bilinear forms and by $\operatorname{Sym}(V \times V, \mathbb{R})$ its subspace that consists of all symmetric bilinear forms.

The Kulkarni-Nomizu product of two bilinear forms $\phi, \psi \in \operatorname{Hom}(V \times V, \mathbb{R})$ is the $(0,4)$-tensor $\phi \otimes \psi: V \times V \times V \times V \rightarrow \mathbb{R}$ defined by

$$
\begin{aligned}
\phi \otimes \psi\left(x_{1}, x_{2}, x_{3}, x_{4}\right)= & \phi\left(x_{1}, x_{3}\right) \psi\left(x_{2}, x_{4}\right)+\phi\left(x_{2}, x_{4}\right) \psi\left(x_{1}, x_{3}\right) \\
& -\phi\left(x_{1}, x_{4}\right) \psi\left(x_{2}, x_{3}\right)-\phi\left(x_{2}, x_{3}\right) \psi\left(x_{1}, x_{4}\right) .
\end{aligned}
$$

Let $W$ be a finite dimensional real vector space equipped with a nondegenerate inner product $\langle\cdot, \cdot\rangle_{W}$. Using the inner product of $W$, we extend the Kulkarni-Nomizu product to $W$-valued bilinear forms $\beta, \gamma \in \operatorname{Hom}(V \times V, W)$, as the $(0,4)$-tensor $\beta \otimes \gamma: V \times V \times V \times V \rightarrow$ $\mathbb{R}$ defined by

$$
\begin{aligned}
\beta \otimes \gamma\left(x_{1}, x_{2}, x_{3}, x_{4}\right)= & \left\langle\beta\left(x_{1}, x_{3}\right), \gamma\left(x_{2}, x_{4}\right)\right\rangle_{W}-\left\langle\beta\left(x_{1}, x_{4}\right), \gamma\left(x_{2}, x_{3}\right)\right\rangle_{W} \\
& +\left\langle\beta\left(x_{2}, x_{4}\right), \gamma\left(x_{1}, x_{3}\right)\right\rangle_{W}-\left\langle\beta\left(x_{2}, x_{3}\right), \gamma\left(x_{1}, x_{4}\right)\right\rangle_{W} .
\end{aligned}
$$

A bilinear form $\beta \in \operatorname{Hom}(V \times V, W)$ is called flat with respect to the inner product $\langle\cdot, \cdot\rangle_{W}$ of $W$ if

$$
\left\langle\beta\left(x_{1}, x_{3}\right), \beta\left(x_{2}, x_{4}\right)\right\rangle_{W}-\left\langle\beta\left(x_{1}, x_{4}\right), \beta\left(x_{2}, x_{3}\right)\right\rangle_{W}=0
$$

for all $x_{1}, x_{2}, x_{3}, x_{4} \in V$, or equivalently if $\beta \otimes \beta=0$.

Associated to each bilinear form $\beta$ is the nullity space $\mathcal{N}(\beta)$ defined by

$$
\mathcal{N}(\beta)=\{x \in V: \beta(x, y)=0 \text { for all } y \in V\}
$$

We need the following result on flat bilinear forms, which is due to Moore (cf. [15, Proposition 2, p. 93]).

Lemma 7. Let $\beta \in \operatorname{Sym}(V \times V, W)$ be a flat bilinear form with respect to a Lorentzian inner product of $W$. If $\operatorname{dim} V>\operatorname{dim} W$ and $\beta(x, x) \neq 0$ for all non-zero $x \in V$, then there is a non-zero isotropic vector $e \in W$ and a bilinear form $\phi \in \operatorname{Sym}(V \times V, \mathbb{R})$ such that $\operatorname{dim} \mathcal{N}(\beta-e \phi) \geq \operatorname{dim} V-\operatorname{dim} W+2$.

We define the map W: $\operatorname{Sym}(V \times V, \mathbb{R}) \rightarrow \operatorname{Hom}(V \times V \times V \times V, \mathbb{R})$ by

$$
\mathrm{W}(\beta)=\mathrm{R}(\beta)-\mathrm{L}(\beta) \otimes\langle\cdot, \cdot\rangle,
$$

where

$$
\mathrm{R}(\beta)=\frac{1}{2} \beta \otimes \beta, \quad \mathrm{L}(\beta)=\frac{1}{n-2}\left(\operatorname{Ric}(\beta)-\frac{\operatorname{scal}(\beta)}{2(n-1)}\langle\cdot, \cdot\rangle\right)
$$




$$
\operatorname{Ric}(\beta)(x, y)=\operatorname{trace} \operatorname{R}(\beta)(\cdot, x, \cdot, y), \quad x, y \in V \text { and } \operatorname{scal}(\beta)=\operatorname{trace} \operatorname{Ric}(\beta) .
$$

To each $\beta \in \operatorname{Sym}(V \times V, \mathbb{R})$ we assign a self-adjoint endomorphism $\beta^{\sharp}$ of $V$ defined by

$$
\left\langle\beta^{\sharp}(x), y\right\rangle=\beta(x, y), \quad x, y \in V .
$$

Lemma 8. Let $\operatorname{dim} V=n \geq 4$ and $\beta \in \operatorname{Sym}(V \times V, \mathbb{R})$. Then $\mathrm{W}(\beta)=0$ if and only if $\beta^{\sharp}$ has an eigenvalue of multiplicity at least $n-1$.

Proof: $\quad$ Let $\beta \in \operatorname{Sym}(V \times V, \mathbb{R})$ be a symmetric bilinear form such that $\mathbf{W}(\beta)=0$. We endow $\mathbb{R}^{3}$ with the Lorentzian inner product $\langle\langle\cdot, \cdot\rangle\rangle$ given by

$$
\left\langle\left\langle\left(x_{1}, x_{2}, x_{3}\right),\left(y_{1}, y_{2}, y_{3}\right)\right\rangle\right\rangle=x_{1} y_{1}+x_{2} y_{3}+x_{3} y_{2}
$$

and define the symmetric bilinear form $\widetilde{\beta}: V \times V \rightarrow \mathbb{R}^{3}$ by

$$
\widetilde{\beta}(x, y)=(\beta(x, y),\langle x, y\rangle,-\mathrm{L}(\beta)(x, y)) .
$$

Since $\mathbf{W}(\beta)=0$ it follows that $\widetilde{\beta}$ is flat with respect to $\langle\langle\cdot, \cdot\rangle\rangle$. From Lemma 7 , we know that there exists a non-zero isotropic vector $e=\left(t_{1}, t_{2}, t_{3}\right) \in \mathbb{R}^{3}$ and a symmetric bilinear form $\phi: V \times V \rightarrow \mathbb{R}$ such that $\operatorname{dim} \mathcal{N}(\widetilde{\beta}-\phi e) \geq n-1$. By setting $V_{1}=\mathcal{N}(\widetilde{\beta}-e \phi)$, we have that

$$
\widetilde{\beta}(x, y)=\phi(x, y) e
$$

or equivalently

$$
\beta(x, y)=\phi(x, y) t_{1}, \quad\langle x, y\rangle=\phi(x, y) t_{2} \text { and } \mathrm{L}(\beta)(x, y)=-\phi(x, y) t_{3}
$$

for all $x \in V_{1}$ and $y \in V$. Therefore,

$$
\beta(x, y)=\langle x, y\rangle \lambda
$$

for all $x \in V_{1}$ and $y \in V$, where $\lambda=t_{1} / t_{2}$. Hence, $\lambda$ is an eigenvalue of $\beta^{\sharp}$ with multiplicity at least $n-1$.

Conversely, assume that

$$
\beta^{\sharp} e_{i}=\lambda e_{i}, \quad 1 \leq i \leq n-1 \text { and } \beta^{\sharp} e_{n}=\mu e_{n},
$$

where $e_{1}, \ldots, e_{n}$ is an orthonormal basis of $V$. A long but straightforward computation then yields $\mathbf{W}(\beta)=0$.

The following proposition is crucial for the proof of Theorem 1.

Proposition 9. Given $n \geq 4$, there exists a positive constant $\varepsilon(n)$, depending only on $n$, such that the following inequality holds

$$
\|\mathrm{W}(\beta)\|^{2} \geq \varepsilon(n)\left|\operatorname{det} \beta^{\sharp}\right|^{4 / n}
$$


for all $\beta \in \operatorname{Sym}(V \times V, \mathbb{R}) \backslash\left(E_{+} \cup E_{-}\right)$, where $V$ is an $n$-dimensional vector space equipped with a positive definite inner product,

$$
E_{ \pm}=\left\{\beta \in \operatorname{Sym}(V \times V, \mathbb{R}): \mathcal{E}_{ \pm}(\beta) \geq n-1\right\}
$$

and $\mathcal{E}_{+}(\beta)$ (respectively, $\left.\mathcal{E}_{-}(\beta)\right)$ is the number of positive (respectively, negative) eigenvalues of $\beta^{\sharp}$, each one counted with its multiplicity.

Proof: $\quad$ Let $\beta \in \operatorname{Sym}(V \times V, \mathbb{R})$ and let $e_{1}, \ldots, e_{n}$ be an orthonormal basis of $V$ that diagonalizes $\beta^{\sharp}$ with corresponding eigenvalues $\lambda_{1}, \ldots, \lambda_{n}$. By an easy computation we obtain

$$
\|\mathrm{W}(\beta)\|^{2}=4 \sum_{i<j}\left(\lambda_{i} \lambda_{j}-\frac{1}{n-2}\left(\left(\lambda_{i}+\lambda_{j}\right) \sum_{k=1}^{n} \lambda_{k}-\left(\lambda_{i}^{2}+\lambda_{j}^{2}\right)-\frac{\sum_{k \neq l} \lambda_{k} \lambda_{l}}{n-1}\right)\right)^{2} .
$$

We consider the functions $\phi, \psi: \mathbb{R}^{n} \rightarrow \mathbb{R}$ defined by

$\phi(x)=4 \sum_{i<j}\left(x_{i} x_{j}-\frac{1}{n-2}\left(\sigma_{1}(x)\left(x_{i}+x_{j}\right)-\left(x_{i}^{2}+x_{j}^{2}\right)-\frac{2 \sigma_{2}(x)}{n-1}\right)\right)^{2}$ and $\psi(x)=\prod_{i=1}^{n} x_{i}$,

where

$$
\sigma_{1}(x)=\sum_{i=1}^{n} x_{i}, \sigma_{2}(x)=\sum_{i<j} x_{i} x_{j} \text { and } x=\left(x_{1}, \ldots, x_{n}\right) .
$$

In order to prove the desired inequality, it is sufficient to show that there exists a positive constant $\varepsilon(n)$, depending only on $n$, such that

$$
\phi(x) \geq \varepsilon(n) \psi(x) \text { for all } x \in U_{n},
$$

where $U_{n}=\mathbb{R}^{n} \backslash\left(K_{+}^{n} \cup K_{-}^{n}\right)$ and $K_{+}^{n}$ (respectively, $\left.K_{-}^{n}\right)$ is the subset of points in $\mathbb{R}^{n}$ with at least $n-1$ positive (respectively, negative) coordinates.

At first we are going to prove that $\phi$ attains a positive minimum on the level set

$$
\Sigma_{n}=\left\{x \in U_{n}: \psi(x)=\varepsilon\right\},
$$

where $\varepsilon= \pm 1$. Since $\phi\left(\Sigma_{n}\right)$ is bounded from below, there exists a sequence $\left\{z_{m}\right\}$ in $\Sigma_{n}$ such that $\lim _{m \rightarrow \infty} \phi\left(z_{m}\right)=\inf \phi\left(\Sigma_{n}\right) \geq 0$. We write $z_{m}=\rho_{m} a_{m}$, where $\rho_{m}=\left\|z_{m}\right\|$ and $a_{m}$ lies in the unit $(n-1)$-dimensional sphere $\mathbb{S}^{n-1} \subset \mathbb{R}^{n}$.

We claim that $\left\{z_{m}\right\}$ is bounded. Suppose, to the contrary, that there exists a subsequence of $\left\{z_{m}\right\}$, which by abuse of notation is again denoted by $\left\{z_{m}\right\}$, such that $\lim _{m \rightarrow \infty} \rho_{m}=\infty$. Since $a_{m} \in \mathbb{S}^{n-1}$ we may assume, by taking a subsequence if necessary, that $\lim _{m \rightarrow \infty} a_{m}=a \in \mathbb{S}^{n-1}$. 
From the homogeneity of $\phi$ and $\psi$ we obtain

$$
\phi\left(a_{m}\right)=\frac{\phi\left(z_{m}\right)}{\rho_{m}^{4}} \text { and } \rho_{m}=\frac{1}{\left|\psi\left(a_{m}\right)\right|^{1 / n}}
$$

Hence, $\lim _{m \rightarrow \infty} \phi\left(a_{m}\right)=0$ and thus $\phi(a)=0$. Lemma 8 implies that at least $n-1$ coordinates of $a$ are equal. On the other hand, $a_{m} \in U_{n}$ and since $U_{n}$ is closed we have $a \in U_{n}$. Therefore, $n-1$ coordinates of $a$ vanish. After an eventual reenumeration, we may suppose that $a=(\varepsilon, 0, \ldots, 0)$. We set

$$
a_{m}=\left(a_{m, 1}, \ldots, a_{m, n}\right) \text { and } \eta_{m}=\left(\sum_{i=2}^{n} a_{m, i}^{2}\right)^{1 / 2}
$$

Since $\psi\left(a_{m}\right) \neq 0$, we may write

$$
\left(a_{m, 2}, \ldots, a_{m, n}\right)=\eta_{m} \theta_{m}
$$

where

$$
\theta_{m}=\left(\theta_{m, 2}, \ldots, \theta_{m, n}\right)
$$

lies in the unit $(n-2)$-dimensional sphere $\mathbb{S}^{n-2} \subset \mathbb{R}^{n-1}$. Then from $\phi\left(z_{m}\right)=\rho_{m}^{4} \phi\left(a_{m}\right)$ we have that

$$
\phi\left(z_{m}\right) \geq 4 \rho_{m}^{4} \sum_{2 \leq i<j}\left(a_{m, i} a_{m, j}-\frac{1}{n-2}\left(\sigma_{1}\left(a_{m}\right)\left(a_{m, i}+a_{m, j}\right)-\left(a_{m, i}^{2}+a_{m, j}^{2}\right)-\frac{2 \sigma_{2}\left(a_{m}\right)}{n-1}\right)\right)^{2} .
$$

We observe that

$$
\sigma_{1}\left(a_{m}\right)=a_{m, 1}+\eta_{m} \sum_{i=2}^{n} \theta_{m, i} \text { and } \sigma_{2}\left(a_{m}\right)=\eta_{m} \widetilde{\sigma}_{2}\left(\theta_{m}\right)
$$

where

$$
\tilde{\sigma}_{2}\left(\theta_{m}\right)=a_{m, 1} \sum_{j=2}^{n} \theta_{m, j}+\eta_{m} \sum_{2 \leq i<j} \theta_{m, i} \theta_{m, j}
$$

Therefore, we have

$$
\phi\left(z_{m}\right) \geq 4 \rho_{m}^{4} \eta_{m}^{2} \delta_{m}
$$

where

$$
\delta_{m}=\sum_{2 \leq i<j}\left(\eta_{m} \theta_{m, i} \theta_{m, j}-\frac{1}{n-2}\left(\sigma_{1}\left(a_{m}\right)\left(\theta_{m, i}+\theta_{m, j}\right)-\eta_{m}\left(\theta_{m, i}^{2}+\theta_{m, j}^{2}\right)-\frac{2 \widetilde{\sigma}_{2}\left(\theta_{m}\right)}{n-1}\right)\right)^{2} .
$$


Moreover, we obtain

$$
\begin{aligned}
\rho_{m}^{4} \eta_{m}^{2} & =\frac{\eta_{m}^{2}}{\left|\psi\left(a_{m}\right)\right|^{4 / n}} \\
& =\frac{\eta_{m}^{2}}{\left|a_{m, 1}\right|^{4 / n}\left|\prod_{i=2}^{n} a_{m, i}\right|^{4 / n}} \\
& =\frac{1}{\left|a_{m, 1}\right|^{4 / n} \eta_{m}^{\frac{2(n-2)}{n}}\left|\prod_{i=2}^{n} \theta_{m, i}\right|^{4 / n}}
\end{aligned}
$$

By passing if necessary to a subsequence, we may assume that

$$
\lim _{m \rightarrow \infty} \theta_{m}=\left(\bar{\theta}_{2}, \ldots, \bar{\theta}_{n}\right) \in \mathbb{S}^{n-2} .
$$

Clearly the above yields

$$
\lim _{m \rightarrow \infty} \rho_{m}^{4} \eta_{m}^{2}=\infty
$$

Using

$$
\lim _{m \rightarrow \infty} \sigma_{1}\left(a_{m}\right)=\varepsilon \text { and } \lim _{m \rightarrow \infty} \widetilde{\sigma}_{2}\left(\theta_{m}\right)=\varepsilon \sum_{k=2}^{n} \bar{\theta}_{k}
$$

we find that

$$
\lim _{m \rightarrow \infty} \delta_{m}=\frac{1}{(n-2)^{2}} \sum_{2 \leq i<j}\left(\bar{\theta}_{i}+\bar{\theta}_{j}-\frac{2 \sum_{k=2}^{n} \bar{\theta}_{k}}{n-1}\right)^{2} .
$$

We claim that $\lim _{m \rightarrow \infty} \delta_{m} \neq 0$. Arguing indirectly, assume that $\lim _{m \rightarrow \infty} \delta_{m}=0$. This implies that $\bar{\theta}_{2}=\cdots=\bar{\theta}_{n} \neq 0$, which contradicts for large $m$ the fact that $a_{m} \in U_{n}$. Thus, by taking limits in (3) we reach a contradiction and this proves the claim that the sequence $\left\{z_{m}\right\}$ is bounded.

By passing if necessary to a subsequence, we have

$$
\lim _{m \rightarrow \infty} z_{m}=z \in \Sigma_{n}
$$

Since $\Sigma_{n}$ doesn't contain any zeros of $\phi$ it follows that

$$
\min \phi\left(\Sigma_{n}\right)=\lim _{m \rightarrow \infty} \phi\left(z_{m}\right)=\phi(z)>0
$$

Hence the function $\phi$ attains a positive minimum $\varepsilon(n)=\phi(z)$ on $\Sigma_{n}$, which obviously depends only on $n$.

Now, let $x \in U_{n}$. Assume that $\psi(x) \neq 0$ and set $\widetilde{x}=x /|\psi(x)|^{1 / n}$. Clearly $\widetilde{x} \in \Sigma_{n}$ and consequently $\phi(\widetilde{x}) \geq \varepsilon(n)$. Since $\phi$ is homogeneous of degree 4 , the desired inequality is obviously fulfilled. In the case where $\psi(x)=0$, the inequality is trivial.

Remark 10. The constant $\varepsilon(n)$ that appears in Proposition 9 is not computed explicitly here, although one can apply the Lagrange multiplier method to compute it. 


\section{The proofs}

At first we recall some well known facts on the total curvature and how Morse theory provides restrictions on the Betti numbers. Let $f:\left(M^{n}, g\right) \rightarrow \mathbb{R}^{n+k}$ be an isometric immersion of a compact, connected and oriented $n$-dimensional Riemannian manifold into the $(n+k)$-dimensional Euclidean space $\mathbb{R}^{n+k}$ equipped with the usual inner product $\langle\cdot, \cdot\rangle$. The normal bundle of $f$ is given by

$$
N_{f} M=\left\{(p, \xi) \in f^{*}\left(T \mathbb{R}^{n+k}\right): \xi \perp d f_{p}\left(T_{p} M\right)\right\}
$$

and the corresponding unit normal bundle is defined by

$$
U N_{f}=\left\{(p, \xi) \in N_{f} M:\|\xi\|=1\right\},
$$

where $f^{*}\left(T \mathbb{R}^{n+k}\right)$ is the induced bundle of $f$ over $M$.

The generalized Gauss map $\nu: U N_{f} \rightarrow \mathbb{S}^{n+k-1}$ is defined by $\nu(p, \xi)=\xi$, where $\mathbb{S}^{n+k-1}$ is the unit $(n+k-1)$-dimensional sphere of $\mathbb{R}^{n+k}$. For each $u \in \mathbb{S}^{n+k-1}$, we consider the height function $h_{u}: M^{n} \rightarrow \mathbb{R}$ defined by $h_{u}(p)=\langle f(p), u\rangle, p \in M^{n}$. Since $h_{u}$ has a degenerate critical point if and only if $u$ is a critical point of the generalized Gauss map, by Sard's theorem there exists a subset $E \subset \mathbb{S}^{n+k-1}$ of measure zero such that $h_{u}$ is a Morse function for all $u \in \mathbb{S}^{n+k-1} \backslash E$. For each $u \in \mathbb{S}^{n+k-1} \backslash E$, we denote by $\mu_{i}(u)$ the number of critical points of $h_{u}$ of index $i$. We also set $\mu_{i}(u)=0$ for any $u \in E$. Following Kuiper [12], we define the total curvature of index $i$ of $f$ by

$$
\tau_{i}(f)=\frac{1}{\operatorname{Vol}\left(\mathbb{S}^{n+k-1}\right)} \int_{\mathbb{S}^{n+k-1}} \mu_{i}(u) d \mathbb{S},
$$

where $d \mathbb{S}$ denotes the volume element of the sphere $\mathbb{S}^{n+k-1}$.

Let $\beta_{i}\left(M^{n} ; \mathbb{F}\right)=\operatorname{dim}_{\mathbb{F}} H_{i}\left(M^{n} ; \mathbb{F}\right)$ be the $i$-th Betti number of $M^{n}$ over an arbitrary coefficient field $\mathbb{F}$. Then, due to weak Morse inequalities [14, Theorem 5.2, p. 29] we have that $\mu_{i}(u) \geq \beta_{i}\left(M^{n} ; \mathbb{F}\right)$, for all $u \in \mathbb{S}^{n+k-1} \backslash E$. By integrating over $\mathbb{S}^{n+k-1}$, we obtain

$$
\tau_{i}(f) \geq \beta_{i}\left(M^{n} ; \mathbb{F}\right) .
$$

For each $(p, \xi) \in U N_{f}$, we denote by $A_{\xi}$ the shape operator of $f$ in the direction $\xi$ which is given by

$$
g\left(A_{\xi} X, Y\right)=\left\langle\alpha_{f}(X, Y), \xi\right\rangle,
$$

where $X, Y$ are tangent vector fields of $M^{n}$ and $\alpha_{f}$ is the second fundamental form of the immersion $f$ viewed as a section of the vector bundle $\operatorname{Hom}\left(T M \times T M, N_{f} M\right)$. There is a natural volume element $d \Sigma$ on the unit normal bundle $U N_{f}$. In fact, if $d V$ is a $(k-1)$-form on $U N_{f}$ such that its restriction to a fiber of the unit normal bundle at $(p, \xi)$ is the volume element of the unit $(k-1)$-sphere of the normal space of $f$ at $p$, then $d \Sigma=d M_{g} \wedge d V$, where $d M_{g}$ is the volume element of $M^{n}$ with respect to the metric $g$. 
Shiohama and $\mathrm{Xu}$ considered for each $0 \leq i \leq n$ the subset $U^{i} N_{f}$ of the unit normal bundle of $f$ defined by

$$
U^{i} N_{f}=\left\{(p, \xi) \in U N_{f}: \operatorname{Index}\left(A_{\xi}\right)=i\right\}
$$

and proved (cf. [18, Lemma, p. 381]) that

$$
\int_{U^{i} N_{f}}\left|\operatorname{det} A_{\xi}\right| d \Sigma=\int_{\mathbb{S}^{n+k-1}} \mu_{i}(u) d \mathbb{S} .
$$

We recall that the $(0,4)$-Riemann curvature tensor $R_{g}$ of $\left(M^{n}, g\right)$ is related to the second fundamental form of $f$ via the Gauss equation

$$
R_{g}(X, Y, Z, W)=\left\langle\alpha_{f}(X, Z), \alpha_{f}(Y, W)\right\rangle-\left\langle\alpha_{f}(X, W), \alpha_{f}(Y, Z)\right\rangle,
$$

where $X, Y, Z, W$ are tangent vector fields of $M^{n}$. In terms of the Kulkarni-Nomizu product, the Gauss equation is written equivalently as

$$
R_{g}=\frac{1}{2} \alpha_{f} \otimes \alpha_{f}
$$

On the other hand, $R_{g}$ admits the following orthogonal decomposition (cf. [11, Chapter 8D])

$$
R_{g}=\mathcal{W}_{g}+\frac{\mathrm{scal}_{g}}{2 n(n-1)} g \otimes g+\frac{1}{n-2}\left(\mathrm{Ric}_{g}-\frac{\mathrm{scal}_{g}}{n} g\right) \otimes g,
$$

which is in addition irreducible with respect to the (simultaneous) action of the orthogonal group $\mathrm{O}(n)$ on the four arguments of $R_{g}$. Equation (6) is equivalent to

$$
R_{g}=\mathcal{W}_{g}+\mathcal{L}_{g} \otimes g
$$

where

$$
\mathcal{L}_{g}=\frac{1}{n-2}\left(\operatorname{Ric}_{g}-\frac{\mathrm{scal}_{g}}{2(n-1)} g\right) .
$$

The $(0,4)$-tensor $\mathcal{W}_{g}$ is the Weyl tensor and the $(0,2)$-tensor $\mathcal{L}_{g}$ is the Schouten tensor of the Riemannian manifold $\left(M^{n}, g\right)$.

Proof of Theorem 1: Let $f:\left(M^{n}, g\right) \rightarrow \mathbb{R}^{n+1}$ be a conformal immersion with unit normal bundle $U N_{f}$ and shape operator $A_{\xi}$ with respect to $\xi$, where $(p, \xi) \in U N_{f}$. Using the Gauss equation, it follows that the Weyl tensor $\mathcal{W}_{\tilde{g}}$ with respect to the metric $\tilde{g}$ induced by $f$ is given by

$$
\mathcal{W}_{\tilde{g}}(p)=\mathbf{W}\left(\alpha_{f}(p)\right)
$$

From Proposition 9 we obtain

$$
\left\|\mathcal{W}_{\tilde{g}}(p)\right\|^{2} \geq \varepsilon(n)\left|\operatorname{det} A_{\xi}(p)\right|^{4 / n}
$$


for all $(p, \xi) \in U^{i} N_{f}$ and $2 \leq i \leq n-2$. By integrating over $U N_{f}$, we have that

$$
\int_{U N_{f}}\left\|\mathcal{W}_{\tilde{g}}\right\|^{n / 2} d \Sigma \geq(\varepsilon(n))^{n / 4} \sum_{i=2}^{n-2} \int_{U^{i} N_{f}}\left|\operatorname{det} A_{\xi}\right| d \Sigma
$$

Using (5), it follows that

$$
\sum_{i=2}^{n-2} \int_{U^{i} N_{f}}\left|\operatorname{det} A_{\xi}\right| d \Sigma=\sum_{i=2}^{n-2} \int_{\mathbb{S}^{n}} \mu_{i}(u) d \mathbb{S}=\operatorname{Vol}\left(\mathbb{S}^{n}\right) \sum_{i=2}^{n-2} \tau_{i}(f)
$$

where $d \mathbb{S}$ is the volume element of the unit $n$-dimensional sphere $\mathbb{S}^{n}$. Therefore, from (8) and bearing in mind (4), we obtain

$$
\int_{U N_{f}}\left\|\mathcal{W}_{\tilde{g}}\right\|^{n / 2} d \Sigma \geq(\varepsilon(n))^{n / 4} \operatorname{Vol}\left(\mathbb{S}^{n}\right) \sum_{i=2}^{n-2} \tau_{i}(f) \geq(\varepsilon(n))^{n / 4} \operatorname{Vol}\left(\mathbb{S}^{n}\right) \sum_{i=2}^{n-2} \beta_{i}\left(M^{n} ; \mathbb{F}\right) .
$$

Observe that

$$
\int_{M^{n}}\left\|\mathcal{W}_{\tilde{g}}\right\|^{n / 2} d M_{\tilde{g}}=\frac{1}{2} \int_{U N_{f}}\left\|\mathcal{W}_{\tilde{g}}\right\|^{n / 2} d \Sigma
$$

Thus, from (9) and the fact that the $L^{n / 2}$-norm of the Weyl tensor is conformally invariant, we have that

$$
\int_{M^{n}}\left\|\mathcal{W}_{g}\right\|^{n / 2} d M_{g} \geq c(n) \sum_{i=2}^{n-2} \beta_{i}\left(M^{n} ; \mathbb{F}\right)
$$

where the constant $c(n)$ is given by $c(n)=(\varepsilon(n))^{n / 4} \operatorname{Vol}\left(\mathbb{S}^{n}\right) / 2$.

Now, assume that

$$
\int_{M^{n}}\left\|\mathcal{W}_{g}\right\|^{n / 2} d M_{g}<c(n)
$$

Then, it follows from $(9)$ that $\sum_{i=2}^{n-2} \tau_{i}(f)<1$. Thus, there exists $u \in \mathbb{S}^{n}$ such that the height function $h_{u}: M^{n} \rightarrow \mathbb{R}$ is a Morse function whose number of critical points of index $i$ satisfies $\mu_{i}(u)=0$ for any $2 \leq i \leq n-2$. From the fundamental theorem of Morse theory (cf. [14, Theorem 3.5, p. 20] or [4, Theorem 4.10, p. 84]), it follows that $M^{n}$ has the homotopy type of a CW-complex with no cells of dimension $i$ for $2 \leq i \leq n-2$. Hence, the homology groups satisfy $H_{i}\left(M^{n} ; \mathbb{Z}\right)=0$ for all $2 \leq i \leq n-2$. Moreover, since there are no 2-cells, we conclude by the cellular approximation theorem that the inclusion of the 1-skeleton $\mathrm{X}^{(1)} \hookrightarrow M^{n}$ induces isomorphism between the fundamental groups. Therefore, the fundamental group $\pi_{1}\left(M^{n}\right)$ is a free group on $\beta_{1}\left(M^{n} ; \mathbb{Z}\right)$ elements and $H_{1}\left(M^{n} ; \mathbb{Z}\right)$ is a free abelian group on $\beta_{1}\left(M^{n} ; \mathbb{Z}\right)$ generators. In particular, if $\pi_{1}\left(M^{n}\right)$ is finite, then $\pi_{1}\left(M^{n}\right)=0$ and hence $H_{1}\left(M^{n} ; \mathbb{Z}\right)=0$. From Poincaré duality and the universal coefficient theorem it follows that $H_{n-1}\left(M^{n} ; \mathbb{Z}\right)=0$. Thus, $M^{n}$ is a simply connected homology sphere and hence a homotopy sphere. By the generalized Poincaré conjecture (Smale $n \geq 5$, Freedman $n=4$ ) we deduce that $M^{n}$ is homeomorphic to $\mathbb{S}^{n}$. 
Proof of Theorem 4: Let $f:\left(M^{n}, g\right) \rightarrow \mathbb{S}^{n+1}$ be an isometric minimal immersion of a compact $n$-dimensional Riemannian manifold, with shape operator $A$. Since $f$ is minimal, the Ricci tensor of $M^{n}$ is given by

$$
\operatorname{Ric}_{g}(X, Y)=(n-1) g(X, Y)-g\left(A^{2} X, Y\right), \quad X, Y \in T M .
$$

Using the Gauss equation and (6), we obtain that

$$
\left\|\mathcal{W}_{g}\right\|^{2}=4 \sum_{i<j}\left(\lambda_{i} \lambda_{j}+\frac{\lambda_{i}^{2}+\lambda_{j}^{2}}{n-2}-\frac{\|A\|^{2}}{(n-1)(n-2)}\right)^{2}
$$

where $\lambda_{1}, \ldots, \lambda_{n}$ are the principal curvatures of $f$. After a straightforward computation, we find that

$$
\left\|\mathcal{W}_{g}\right\|^{2}=\gamma(n)\|A\|^{4}-\delta(n)\left\|\operatorname{Ric}_{g}-(n-1) g\right\|^{2}
$$

where

$$
\gamma(n)=\frac{2\left(n^{2}-3 n+5\right)}{(n-1)(n-2)} \text { and } \delta(n)=\frac{2(n+1)}{n-2} .
$$

From the Cauchy-Schwartz inequality we have that

$$
\|A\|^{4} \leq n\left\|A^{2}\right\|^{2}=n\left\|\operatorname{Ric}_{g}-(n-1) g\right\|^{2} .
$$

Therefore, from (10) we obtain

$$
\left\|\mathcal{W}_{g}\right\|^{2} \leq a(n)\left\|\operatorname{Ric}_{g}-(n-1) g\right\|^{2}
$$

where $a(n)=n \gamma(n)-\delta(n)$. By integrating over $M^{n}$, it follows that

$$
\int_{M^{n}}\left\|\mathcal{W}_{g}\right\|^{n / 2} d M_{g} \leq(a(n))^{n / 4} \int_{M^{n}}\left\|\operatorname{Ric}_{g}-(n-1) g\right\|^{n / 2} d M_{g}
$$

The proof follows from (11), Theorem 1 applied to the composition of $f$ with the stereographic projection and the fact that the $L^{n / 2}$-norm of the Weyl tensor is conformally invariant. Clearly, $c_{1}(n)=c(n) /(a(n))^{n / 4}$.

Proof of Theorem 6: Assume that $\left(M^{n}, g\right)$ admits an isometric immersion into the sphere $\mathbb{S}^{n+1}$ or the hyperbolic space $\mathbb{H}^{n+1}$. Using the orthogonal decomposition of the curvature tensor $R_{g}$ in (6) and recalling the fact that $R_{1}=(1 / 2) g \otimes g$, we obtain

$$
\left\|R_{g}-\frac{\mathrm{scal}_{g}}{n(n-1)} R_{1}\right\|^{2}=\left\|\mathcal{W}_{g}\right\|^{2}+\left\|\frac{1}{n-2}\left(\operatorname{Ric}_{g}-\frac{\mathrm{scal}_{g}}{n} g\right) \otimes g\right\|^{2} \geq\left\|\mathcal{W}_{g}\right\|^{2}
$$

Thus, it follows that

$$
\int_{M^{n}}\left\|R_{g}-\frac{\operatorname{scal}_{g}}{n(n-1)} R_{1}\right\|^{n / 2} d M_{g} \geq \int_{M^{n}}\left\|\mathcal{W}_{g}\right\|^{n / 2} d M_{g}
$$

Now the proof follows from (12), Theorem 1 and Remark 2. 


\section{References}

[1] K. Akutagawa, B. Botvinnik, O. Kobayashi, and H. Seshadri, The Weyl functional near the Yamabe invariant, J. Geom. Anal. 13 (2003), no. 1, 1-20, DOI 10.1007/BF02930992. MR1967032

[2] E. Cartan, La déformation des hypersurfaces dans l'espace conforme réel à $n \geq 5$ dimensions, Bull. Soc. Math. France 45 (1917), 57-121 (French). MR1504762

[3] G. Catino, On conformally flat manifolds with constant positive scalar curvature, Proc. Amer. Math. Soc. 144 (2016), no. 6, 2627-2634, DOI 10.1090/proc/12925. MR3477081

[4] J. Cheeger and D.G. Ebin, Comparison theorems in Riemannian geometry, North-Holland Publishing Co., Amsterdam-Oxford; American Elsevier Publishing Co., Inc., New York, 1975. North-Holland Mathematical Library, Vol. 9. MR0458335

[5] S.S. Chern and J. Simons, Characteristic forms and geometric invariants, Ann. of Math. (2) 99 (1974), 48-69. MR0353327

[6] M. Dajczer and L.A. Florit, On conformally flat submanifolds, Comm. Anal. Geom. 4 (1996), no. 1-2, 261-284, DOI 10.4310/CAG.1996.v4.n2.a3. MR1393564

[7] M. do Carmo, M. Dajczer, and F. Mercuri, Compact conformally flat hypersurfaces, Trans. Amer. Math. Soc. 288 (1985), no. 1, 189-203, DOI 10.2307/2000435. MR773056

[8] M.P. Dussan and M.H. Noronha, Manifolds with 2-nonnegative Ricci operator, Pacific J. Math. 204 (2002), no. 2, 319-334, DOI 10.2140/pjm.2002.204.319. MR1907895

[9] M.J. Gursky, The Weyl functional, de Rham cohomology, and Kähler-Einstein metrics, Ann. of Math. (2) 148 (1998), no. 1, 315-337, DOI 10.2307/120996. MR1652920

[10] M. Itoh and H. Satoh, Isolation of the Weyl conformal tensor for Einstein manifolds, Proc. Japan Acad. Ser. A Math. Sci. 78 (2002), no. 7, 140-142. MR1930219

[11] W. Kühnel, Differential geometry, Student Mathematical Library, vol. 77, American Mathematical Society, Providence, RI, 2015. Curves - surfaces - manifolds; Third edition [of MR1882174]; Translated from the 2013 German edition by Bruce Hunt, with corrections and additions by the author. MR3443721

[12] N.H. Kuiper, Minimal total absolute curvature for immersions, Invent. Math. 10 (1970), 209-238. MR0267597

[13] M. Listing, $L^{n / 2}$-curvature gaps of the Weyl tensor, J. Geom. Anal. 24 (2014), no. 2, 786-797, DOI 10.1007/s12220-012-9356-7. MR3192298

[14] J. Milnor, Morse theory, Based on lecture notes by M. Spivak and R. Wells. Annals of Mathematics Studies, No. 51, Princeton University Press, Princeton, N.J., 1963. MR0163331

[15] J.D. Moore, Conformally flat submanifolds of Euclidean space, Math. Ann. 225 (1977), no. 1, 89-97. MR0431046

[16] U. Pinkall, Compact conformally flat hypersurfaces, Conformal geometry (Bonn, 1985/1986), Aspects Math., E12, Vieweg, Braunschweig, 1988, pp. 217-236. MR979794

[17] H. Seshadri, Weyl curvature and the Euler characteristic in dimension four, Differential Geom. Appl. 24 (2006), no. 2, 172-177, DOI 10.1016/j.difgeo.2005.08.008. MR2198792

[18] K. Shiohama and H. Xu, Lower bound for $L^{n / 2}$ curvature norm and its application, J. Geom. Anal. 7 (1997), no. 3, 377-386, DOI 10.1007/BF02921626. MR1674797 
[19] _ Rigidity and sphere theorems for submanifolds, Kyushu J. Math. 48 (1994), 291-306.

[20] _ Rigidity and sphere theorems for submanifolds II, Kyushu J. Math. 54 (2000), 103-109.

[21] M.A. Singer, Positive Einstein metrics with small $L^{n / 2}$-norm of the Weyl tensor, Differential Geom. Appl. 2 (1992), no. 3, 269-274, DOI 10.1016/0926-2245(92)90014-E. MR1245327

[22] Th. Vlachos, Integral curvature and topological obstructions for submanifolds, Geom. Dedicata 166 (2013), 289-305.

Department of Mathematics, University of Ioannina, 45110 Ioannina, Greece E-mail addresses: tvlachos@uoi.gr, chonti@cc.uoi.gr 